\title{
A HOMOTOPY CLASSIFICATION OF 2-COMPLEXES WITH FINITE CYCLIC FUNDAMENTAL GROUP
}

\author{
BY MICHEAL N. DYER AND ALLAN J. SIERADSKI \\ Communicated by M. L. Curtis, June 5, 1972
}

For an arbitrary positive integer $n$, let $Z_{n}$ denote the cyclic group of order $n$, and let $P_{n}=S^{1} \cup_{n} e^{2}$ be the pseudo-projective plane of order $n$.

THEOREM. Let $X$ be a connected finite 2-dimensional $\mathrm{CW}$-complex with fundamental group $Z_{n}$. Then

(1) $X$ has the homotopy type of the sum $P_{n} \vee S^{2} \vee \cdots \vee S^{2}$ of the pseudoprojective plane $P_{n}$ and rank $\mathrm{H}_{2}(X)$-copies of the 2-sphere $\mathrm{S}^{2}$.

(2) There is a homotopy equivalence $f: X \rightarrow P_{n} \vee S^{2} \vee \cdots \vee S^{2}$ realizing any prescribed Whitehead torsion $\tau(f) \in \mathrm{Wh}\left(Z_{n}\right)$.

The result (1) was established in the prime order case by W. H. Cockcroft and R. G. Swan [3]. The work of P. Olum on the self-equivalences of the pseudo-projective plane $P_{n}([6],[7])$ shows that every element of the Whitehead group Wh $\left(Z_{n}\right)$ is realized as the torsion of some self-equivalence $P_{n} \rightarrow P_{n}$, so that (2) is a consequence of (1).

COROLlary. For connected finite 2-dimensional $\mathrm{CW}$-complexes with finite cyclic fundamental group, homotopy type and simple homotopy type coincide.

This generalizes to the nonprime order case a recent observation of W. H. Cockcroft and R. M. F. Moss [2].

Sketch of a Proof of the TheORem. Each CW-complex under consideration has the simple homotopy type of a complex $P$ that is modeled in an obvious fashion on some presentation $\mathscr{P}=\left\langle a_{1}, \ldots, a_{k}\right.$ : $\left.r_{1}, \ldots, r_{m}\right\rangle(m \geqq k)$ of the cyclic group $Z_{n}$. There are Nielsen transformations which reduce such a presentation to one of pre-Abelian form [5, p. 140]

$$
\mathscr{Q}=\left\langle b_{1}, \ldots, b_{k}: b_{1} W_{1}, \ldots, b_{k-1} W_{k-1}, b_{k}^{n} W_{k}, W_{k+1}, \ldots, W_{m}\right\rangle,
$$

where the exponent sum of each word $W_{i}$ with respect to each generator $b_{j}$ is zero. Moreover, this Nielsen reduction $\mathscr{P} \rightarrow \mathscr{Q}$ corresponds to a simple homotopy equivalence $P \rightarrow Q$ of the associated topological models. Associated with each topological model $P$ of a presentation $\mathscr{P}$ is the cellular chain complex $C_{*}(\widetilde{P})$ of its universal covering $\widetilde{P}$; the chain groups are free $Z_{n}$-modules which we give preferred bases according to a

AMS (MOS) subject classifications (1969). Primary 5540.

Copyright (C) American Mathematical Society 1973 
specific natural system. The chain complex $C_{*}=C_{*}(\tilde{Q})$ with its preferred bases is

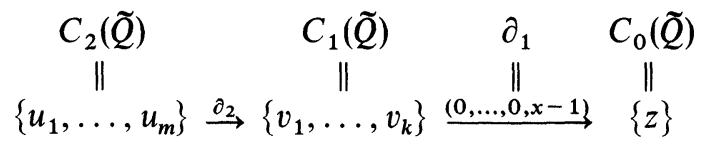

where $\{, \ldots$,$\} is the free Z_{n}$-module with the enclosed basis, and $x$ is the generator of the multiplicative cyclic group $Z_{n}$.

Using Jacobinski's cancellation theorem for projective $Z_{n}$-modules ([4], [8, p. 215], [9, p. 178]), it is possible to choose a new basis $w_{1}, \ldots, w_{m}$ for the chain group $C_{2}=C_{2}(\widetilde{Q})$ such that the matrix of the boundary operation $\partial_{2}: C_{2}(\tilde{Q}) \rightarrow C_{1}(\tilde{Q})$ with respect to this new basis for $C_{2}$ and the old basis $v_{1}, \ldots, v_{k}$ for $C_{1}$ is

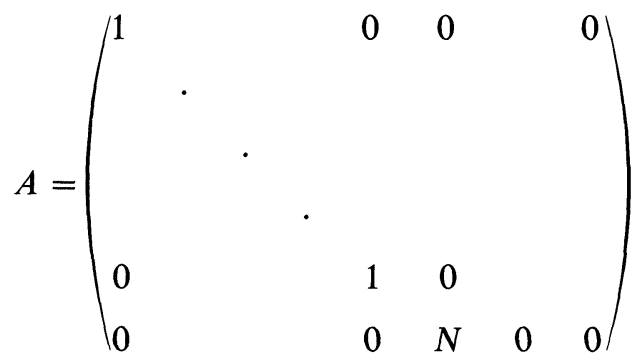

where the identity block is a $(k-1) \times(k-1)$ matrix and where $N=$ $1+x+\cdots+x^{n-1}$ is in the integral group ring of $Z_{n}$. The chain complex $C_{*}$ with the new preferred basis for $C_{2}$ takes the form

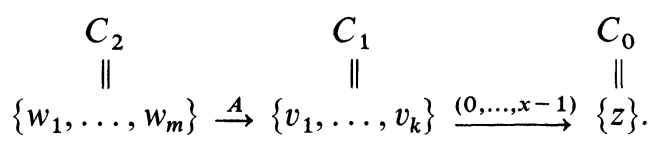

With these preferred bases, the chain complex $C_{*}$ is realizable as the cellular chain complex $C_{*}(\widetilde{R})$ of the universal covering $\widetilde{R}$ of the complex $R$ modeled on the presentation $\mathscr{R}=\left\langle c_{1}, \ldots, c_{k}: c_{1}, \ldots, c_{k-1}, c_{k}^{n}, 1, \ldots, 1\right\rangle$ with $m-k$ trivial relators. The identity map between the chain complexes $C_{*}(\widetilde{R})$ and $C_{*}(\widetilde{Q})$ can be realized by a map $f: R \rightarrow Q$ that is necessarily a homotopy equivalence. This completes the proof of the theorem since the space $R$ modeled on the presentation $\mathscr{R}$ has the simple homotopy type of the sum $P_{n} \vee S^{2} \vee \cdots \vee S^{2}$ of the pseudo-projective plane $P_{n}$ and $m-k$ copies of the 2 -sphere $S^{2}$.

Full details of these and related results will appear elsewhere.

\section{BIBLIOGRAPHY}

1. H. Bass, Algebraic K-theory, Benjamin, New York, 1968. MR 40 \# 2736. 
2. W. H. Cockcroft and R. M. F. Moss, On the homotopy type of certain two-dimensional complexes, Proc. London Math. Soc. (3) 11 (1961), 193-202.

3. W. H. Cockcroft and R. G. Swan, On the homotopy type of certain two-dimensional complexes, Proc. London Math. Soc. (3) 11 (1961), 194-202. MR 23 \# A3567.

4. H. Jacobinski, Genera and decompositions of lattices over orders, Acta. Math. 121 (1968), 1-29. MR 40 \# 4294.

5. W. Magnus, A. Karrass and D. Solitar, Combinatorial group theory: Presentations of groups in terms of generators and relations, Pure and Appl. Math., vol. 13, Interscience, New York, 1966. MR 34 \# 7617.

6. P. Olum, Self-equivalences of pseudo-projective planes, Topology 4 (1965), 109-127. MR 31 \# 2725.

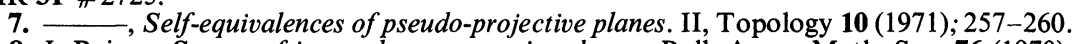

8. I. Reiner, Survey of integral representation theory, Bull. Amer. Math. Soc. 76 (1970), 159-227. MR 40 \# 7302.

9. R. Swan, K-theory of finite groups and orders, Lecture Notes in Math., vol. 149, SpringerVerlag, Berlin and New York, 1970.

Department of Mathematics, University of Oregon, Eugene, Oregon 97403 